\title{
Disturbance Observer based Boundary Tracking for Environment Monitoring
}

\author{
J.-S. Kim*, P. P. Menon**, J. Back ${ }^{\dagger}$ and H. Shim*** \begin{abstract}
the boundary tracking problem can be reformulated into a robust control of uncertain double integrator first. Then, a disturbance observer (DOB) based control is proposed solving the robust control problem. Unlike the existing results in the literature, the proposed DOB based control requires only the local position measurement of the boundary (not the gradient information). The performance of the proposed control is demonstrated for two cases: the measurement of the boundary is given in a continuous or discrete manner. Finally, it is shown that the proposed control can be used for environmental monitoring as well by showing that the agent follows a level curve of real environmental monitoring data.
\end{abstract} \\ Abstract - This paper presents a boundary tracking control of an agent. To this end, it is shown that
}

Keywords: Boundary tracking, Disturbance observer, Robust control, Environmental monitoring

\section{Introduction}

Spiraling technique [1], UUV-gas algorithm [2], and Snake algorithm [3] have been applied to track perimeter of regions of interest. Hybrid coordination algorithms, which are primarily decentralized, have been investigated for tracking moving boundaries in [4].

In [5], a single agent tracks a scalar potential field using a reactive control law that has roots on the isoline following control methodology. An assumption is on the availability of the gradient information of the scalar field. Extremum seeking algorithms are proposed in [6] for source localisation, which is a different problem to the one considered in the paper, and where authors estimate gradient by probing scalar field periodically.

Spatial gradient direction is estimated using a team of vehicles in [7], even in the presence of constrained communication and using Poisson integrals method in [8]. These spatial gradient estimation techniques are of use to device separate control ideas for steering vehicles along the boundary. A boundary tracking control law based on sub-optimal sliding mode techniques which require only instantaneous contaminant density level measurement has been developed in [9] and has been validated using real data from the Eyjafyallajökull volcanic eruption.

In [10], a bio-inspired technique from the behavior of

$\dagger$ Corresponding Author: School of Robotics, Kwangwoon University, Korea. (backhoon@kw.ac.kr)

* Dept. of EIE, Seoul National University of Science and Technology, Korea. (jungsu@seoultech.ac.kr)

** College of Engineering, Mathematics and Physical Sciences, University of Exeter, Exeter, United Kingdom.

(P.M.Prathyush@exeter.ac.uk)

*** Automation and Systems Research Institute (ASRI) and Dept. of Electrical and Computer Engineering, Seoul National University, Korea. (hshim@snu.ac.kr.)

Received: October 11, 2016; Accepted: February 17, 2017 fireflies/glowworms, which was originally proposed in [11] has been extended to carry out mapping of two dimensional boundaries and subsequently to map three dimensional boundaries [12].

The objective of this paper is to design a feedback control law making an agent follow a boundary which is provided by either a function of the agent's position or a level set of real measured data (e.g. leakage of harmful gas, nuclear radiation from unshielded devices, oil spill in oceans, spread of ash clouds from volcanic eruptions) given in a discrete manner.

To achieve the objective, the contribution of the paper is threefold. First, it is shown that the boundary tracking problem is equivalent to an output zeroing problem of an uncertain double integrator. This means that the boundary tracking is achieved by designing a robust control. Second, an output feedback robust control is designed to solve the reformulated problem using only measurable signal. Third, it is demonstrated that the proposed boundary tracking can be applied to environmental monitoring.

In order to design the robust control, a disturbance observer (DOB) based control is devised for the uncertain double integrator under the assumption that only the boundary information at the agent's position is measurable [13]. The disturbance observer estimates whole system uncertainties and the estimated uncertainty is used to cancel out the uncertainties. Besides, the high-gain observer type Q-filter in the DOB estimates not only the error between the current agent's position and the boundary but also its derivative. These estimates are used in the controller in order to stabilize the nominal model. Note that this estimated derivative makes a sharp difference between the proposed scheme and the existing result in which the derivative information is assumed to be available to the controller. In other words, thanks to the 
estimated derivative information, the proposed control works using only measurable tracking error.

The designed DOB based control is applied to two examples. In the first example, the measurement is given continuously. On the other hand, the measurement is provided in a discrete manner in the second example. In both examples, it is shown that the proposed DOB based control achieves boundary tracking successfully. Finally, the performance of the proposed control is demonstrated by applying it to the tracking of a boundary made by environmental monitoring data which is provided discretely.

\section{Preliminary: Disturbance Observer}

In order to present the controller design methodology, this section introduces a disturbance observer based robust control design reported in $[13,14]$.

Consider the disturbance observer (DOB) based control systems depicted in Fig. 1. The uncertain plant is described by

$$
\begin{aligned}
& \dot{x}_{i}=x_{i+1}, \quad i=1, \ldots, n-1 \\
& \dot{x}_{n}=\phi_{1} x_{1}+\phi_{2} x_{2}+\cdots+\phi_{n} x_{n}+g(u+d) \\
& y=x_{1},
\end{aligned}
$$

where $\phi_{i}(i=1, \cdots, n)$ and $g$ are uncertain parameters and $d$ denotes unknown bounded disturbance. The corresponding nominal model of the uncertain system is assumed to be given by

$$
\begin{aligned}
& \dot{\bar{x}}_{i}=\bar{x}_{i+1}, \quad i=1, \ldots, n-1 \\
& \dot{\bar{x}}_{n}=\bar{\phi}_{1} \bar{x}_{1}+\bar{\phi}_{2} \bar{x}_{2}+\cdots+\bar{\phi}_{n} \bar{x}_{n}+\bar{g} u_{r} \\
& \bar{y}=\bar{x}_{1},
\end{aligned}
$$

where $\bar{\phi}_{i}(i=1, \cdots, n)$ and $\bar{g}$ are known nominal parameters. In the DOB based control design, it is assumed that the nominal control $u_{r}$ for the nominal model is properly designed such that

$$
\lim _{t \rightarrow \infty} \bar{y}(t)=y_{r e f}
$$

where $y_{\text {ref }}$ is the output reference. In other words, if there are no parameter uncertainties and external disturbances, control $u_{r}$ achieves perfect output tracking. On the top of this nominal controller, a DOB is designed in order to estimate and reject the undesired effect of uncertainties in the system. For notational simplicity, denote the transfer function of the uncertain system by $P(s)$, that of the nominal model by $P_{n}(s)$, and those $Q$-filters by $Q_{A}(s)$ and $Q_{B}(s)$. Using these notations, the state space models of the DOB are given by

$$
\begin{aligned}
& Q_{B}(s): \quad \dot{p}=A_{\alpha \tau} p+\frac{\alpha_{1}}{\tau^{n}} B u, \\
& u^{+}=p_{1} \\
& P_{n}^{-1}(s) Q_{A}(s): \quad \dot{q}=A_{\alpha \tau} q+B_{\alpha \tau} y, \\
& \hat{u}_{p}=\frac{1}{\bar{g}}\left(\dot{q}_{n}-\bar{\varphi}^{T} q\right) \\
& =-\frac{\alpha_{1}}{\tau^{n}} \frac{1}{\bar{g}}\left(q_{1}-y\right)-\frac{1}{\bar{g}} \bar{\varphi}^{T} q \\
& \hat{d}=\Phi_{a}\left(\hat{u}_{p}-u^{+}\right) \\
& u=u_{r}-\hat{d}
\end{aligned}
$$

where

$$
\begin{aligned}
& A_{\alpha \tau}=\left[\begin{array}{ccccc}
-\frac{\alpha_{n}}{\tau} & 1 & 0 & \cdots & 0 \\
-\frac{\alpha_{n-1}}{\tau} & 0 & 1 & \cdots & 0 \\
\vdots & \vdots & \vdots & \ddots & \vdots \\
-\frac{\alpha_{2}}{\tau^{n-1}} & 0 & \cdots & 0 & 1 \\
-\frac{\alpha_{1}}{\tau^{n}} & 0 & \cdots & 0 & 0
\end{array}\right], B_{\alpha \tau}=\left[\begin{array}{c}
\frac{1}{\tau} \alpha_{n} \\
\vdots \\
\frac{1}{\tau^{n}} \alpha_{1}
\end{array}\right], \\
& B=\left[\begin{array}{c}
0_{n-1} \\
1
\end{array}\right], \bar{\phi}=\left[\begin{array}{c}
\bar{\phi}_{1} \\
\vdots \\
\bar{\phi}_{n}
\end{array}\right],
\end{aligned}
$$

$\tau$ and $\alpha_{i}{ }^{\prime}$ s are design parameters, and $\Phi_{a}($.$) is$ saturation function with saturation bound $a>0$.

The principle of such a DOB design is as follows. In Fig. 1 , the input to the uncertain system $P(s)$ is $u+d$. An intuitive way to estimate $u+d$ is to use the output of the uncertain system $y$ being its input conceptually corresponds to $u+d$. Since $Q_{B}(s)$ is also a stable filter with unity DC gain, $u^{+}$also corresponds to $u$. Hence, the output of $P_{n}^{-1}(s) Q_{A}(s)$, say $\hat{u}_{p}-u^{+}$is an estimate of the lumped disturbance in the system. The saturation function is employed for stability guarantee by avoiding peaking phenomena since, in fact, $Q_{A}$ filter in this DOB is highgain observer. The estimated $\hat{d}$ is used to cancel out the lumped disturbance. An interpretation of this DOB based control is that the DOB with uncertain plant $P(s)$ behaves like $P_{n}(s)$ dynamically, which makes the nominal

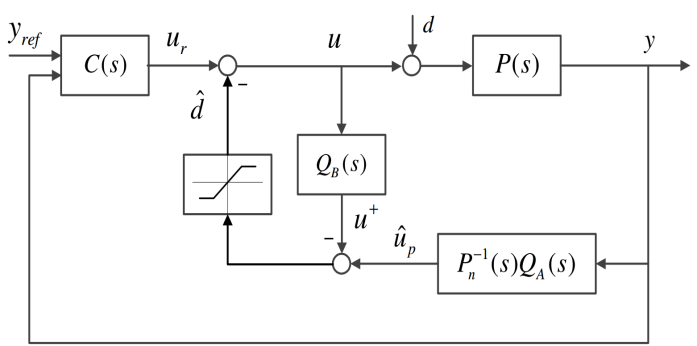

Fig. 1. The basic structure of the disturbance observer [14] 
controller achieve the output tracking successfully even for the uncertain system. One important aspect of this DOB is that the state of filter $Q_{A}(s)$ is state estimate of the uncertain plant $P(s)$ since it is nothing but a highgain observer of the uncertain system. This property plays a key role in designing the boundary tracking control in this paper. For details, see [13].

\section{Main Result: DOB based Boundary Tracking}

\subsection{Problem definition}

Consider the agent dynamics described by

$$
\begin{aligned}
& \dot{x}=V \cos \theta \\
& \dot{y}=V \sin \theta \\
& \dot{\theta}=u
\end{aligned}
$$

where $x$ and $y$ denote the position of the agent and $\theta$ the heading direction of the agent. In addition, $V$ is the agent's velocity which is assumed to be constant. For details, see Fig. 2.

The objective of the paper is to design a feedback control law for the agent such that the agent follows the trajectory defined by

$$
D=\left\{(x, y) \in R^{2} \mid \gamma(x, y)=\gamma^{*}\right\}
$$

Where $\gamma(x, y)$ stands for a function describing physical value at position $(x, y)$, and $\gamma^{*}$ is a given and known constant.

Assumption 1: $x(t)$ and $y(t)$ belong to a compact set for all $t \geq 0$. The function $\gamma(x, y)$ itself is unknown but only its value at $(x, y)$ is available to the agent when the agent is located at position $(x, y)$. Besides, $\gamma(x, y)$ is continuously differentiable and $\frac{d\|\nabla \gamma(x, y)\|}{d t}$ is bounded.

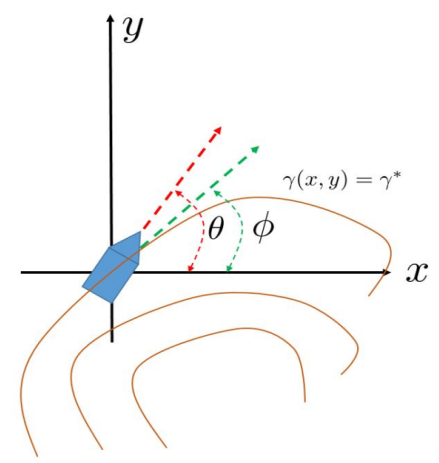

Fig. 2. Coordinates for boundary tracking problem [9]

\subsection{DOB based boundary tracking}

This subsection shows that the problem of the boundary tracking is actually a robust control design, and presents a DOB based boundary tracking controller.

For the purpose of reformulating the boundary control design into a robust control design, define a new variable given by

$$
\sigma(t)=\gamma(x(t), y(t))-\gamma^{*} \in R
$$

From the definition, variable $\sigma(t)$ can be understood as tracking error. Then, the time derivative of the variable $\sigma(t)$ along the agent dynamics is given by

$$
\begin{aligned}
\dot{\sigma}(t) & =V \frac{\partial \gamma}{\partial x} \cos \theta+V \frac{\partial \gamma}{\partial y} \sin \theta \\
& =V\|\nabla \gamma\| \sin (\theta-\varphi), \\
\ddot{\sigma}(t) & =\underbrace{V \frac{d\|\nabla \gamma(x, y)\|}{d t} \sin (\theta-\varphi)}_{\equiv \xi(t)} \\
+\underbrace{V\|\nabla \gamma(x, y)\| \cos (\theta-\varphi)(\dot{\theta}-\dot{\varphi})}_{\equiv b(t)} & \underbrace{\xi \xi(t)+b(t)(u(t)-\dot{\varphi}(t))} \\
& =b(t) u(t)+\xi(t)-b(t) \dot{\varphi}(t),
\end{aligned}
$$

where $u(t) \equiv \dot{\theta}(t)$ and $\phi$ denotes the angle indicating the tangent line at $\gamma(x, y)$, i.e. $\varphi=\operatorname{atan} 2(y, x)$ (fourquadrant inverse tangent). With these dynamics in mind, define new variables

$$
z_{1}=\sigma(t), \quad z_{2}=\dot{\sigma}(t)
$$

Then, its dynamics is written as

$$
\begin{aligned}
& \dot{z}_{1}=z_{2}(t) \\
& \dot{z}_{2}=b(t) u(t)+\xi(t)-b(t) \dot{\varphi}(t) \\
& y_{z}=z_{1}(=\sigma(t)) .
\end{aligned}
$$

where $y_{z}$ is the measurable output variable. Note that $b(t)$, $\xi(t)$, and $\dot{\varphi}(t)$ are bounded for all $t$ and dependent on $\nabla \gamma(x, y)$ which is unknown. This $z$ system is an uncertain double integrator by viewing $b(t)$ and $\xi(t)-b(t) \dot{\varphi}(t)$ as an uncertain parameter and unknown bounded disturbances, respectively. Hence, the boundary tracking control design boils down to designing a robust output zeroing controller for the uncertain double integrator.

Assumption 2: $b(t)$ satisfies $0<K_{m}<b(t)<K_{M}$ where $K_{m}$ and $K_{M}$ are known constants.

In light of the definition of $\theta$ and $\phi$, positivity of 
$b(t)$ for all $t$ in Assumption 2 means that the agents tracks the trajectory with maintaining $|\theta(t)-\varphi(t)| \leq \theta_{e}<\frac{\pi}{2}$ for a constant $\theta_{e}$ because $\cos (\theta(t)-\varphi(t))>0$. Note that if the boundary to be followed is smooth, the controller is designed properly, and the agent's heading direction is well aligned with $\phi$ initially, this condition is satisfied.

For the sake of designing a DOB based boundary tracking controller, consider the nominal model of the uncertain system (4) as follows

$$
\begin{aligned}
& \dot{\bar{z}}_{1}=\bar{z}_{2}(t) \\
& \dot{\bar{z}}_{2}=\bar{b} u_{r}(t) \\
& \bar{y}_{z}=\bar{z}_{1}(=\bar{\sigma}(t))
\end{aligned}
$$

where $\bar{b}=\frac{K_{m}+K_{M}}{2}$ and $u_{r}$ denotes the control input to the nominal model. In order to design a DOB based controller, the nominal controller is designed as

$$
u_{r}(t)=\frac{1}{\bar{b}}\left(k_{1} q_{1}+k_{2} q_{2}\right)
$$

where $k_{1}$ and $k_{2}$ are determined such that $\left[\begin{array}{ll}0 & 1 \\ k_{1} & k_{2}\end{array}\right]$ is Hurwitz, and $q_{1}$ and $q_{2}$ are the Q-filter state which can be interpreted as a state estimate since the Q-filter is a highgain observer of the uncertain system (4) [15]. Note that if both $z_{1}$ and $z_{2}$ are measurable, they can be used instead of $q_{1}$ and $q_{2}$ in (5). However, since $z_{2}$ is not available in general, its estimate is used in the nominal controller, which is the main advantage of employing the DOB.

According to the DOB design introduced in the Section 2 , the DOB for the uncertain system (4) is given by

$$
\begin{gathered}
\dot{p}=\left[\begin{array}{rr}
-\frac{\alpha_{2}}{\tau} & 1 \\
-\frac{\alpha_{1}}{\tau^{2}} & 0
\end{array}\right] p+\frac{\alpha_{1}}{\tau^{2}}\left[\begin{array}{l}
0 \\
1
\end{array}\right] u \\
u^{+}=p_{1} \\
\dot{q}=\left[\begin{array}{ll}
-\frac{\alpha_{2}}{\tau} & 1 \\
-\frac{\alpha_{1}}{\tau^{2}} & 0
\end{array}\right] q+\left[\begin{array}{c}
\frac{\alpha_{2}}{\tau} \\
\frac{\alpha_{1}}{\tau^{2}}
\end{array}\right] y_{z} \\
\hat{u}_{p}=\frac{1}{\bar{b}} \dot{z}_{2} \quad \\
=\frac{1}{\bar{b}} \dot{q}_{2}=\frac{1}{\bar{b}}\left(\frac{\alpha_{1}}{\tau^{2}}\left(-q_{1}+y_{z}\right)\right),
\end{gathered}
$$

where $\alpha_{1}, \alpha_{2}$ are determined such that $s^{2}+\alpha_{2} s+\alpha_{1}$ becomes a stable polynomial with sufficiently small $\alpha_{1}$ in accordance with the tuning method shown in [13]. The output of the DOB becomes

$$
\hat{d}=\Phi_{a}\left(\hat{u}_{p}-u^{+}\right)
$$

Then, the DOB based boundary tracking control is given by

$$
u=u_{r}-\hat{d}=\frac{1}{\bar{b}}\left(k_{1} q_{1}+k_{2} q_{2}\right)-\hat{d}
$$

In view of the main result of the DOB based control design [13], the proposed boundary tracking control is summarized in the following theorem.

Theorem 1: Suppose that Assumptions 1 and 2 are satisfied. Then, there exists $\tau^{*}>0$ for a given $\varepsilon>0$ such that the DOB based boundary tracking control (6) with any $0<\tau<\tau^{*}$ result in

$$
\lim _{t \rightarrow \infty}|\sigma(t)|=\left|\gamma(x(t), y(t))-\gamma^{*}\right|<\varepsilon .
$$

\section{Application of DOB based Boundary Tracking Control}

In this section, the DOB based boundary tracking control developed in the previous section is applied to two cases: continuous $\gamma($.$) and discrete \gamma($.

\subsection{Continuous measurement case}

Suppose that the agent (1) is required to follow the trajectory (s) with

$$
\gamma(x, y)=x^{2}+y^{2}, \quad \gamma^{*}=4
$$

and that the function value of $\gamma(x, y)$ is available to the agent when the agent is located at the position $(x, y)$. Note that Assumption 1 holds true since it follows from the agent dynamics and

$$
\begin{aligned}
& \|\nabla \gamma\|=2 \sqrt{x^{2}+y^{2}}<\infty, \\
& \frac{d\|\nabla \gamma\|}{d t}=V \frac{2 x \cos \theta+2 y \sin \theta}{\sqrt{x^{2}+y^{2}}}<\infty,
\end{aligned}
$$

with bounded trajectory $\gamma$ and $x^{2}+y^{2} \neq 0$. Moreover, we have

$$
|\xi(t)| \leq 2 V^{2}, \quad|b(t)| \leq 2 V \sqrt{x^{2}+y^{2}}
$$

This means that Assumptions 2 holds true as well. In accordance with the DOB based boundary tracking control in the previous section, the nominal control parameters are 


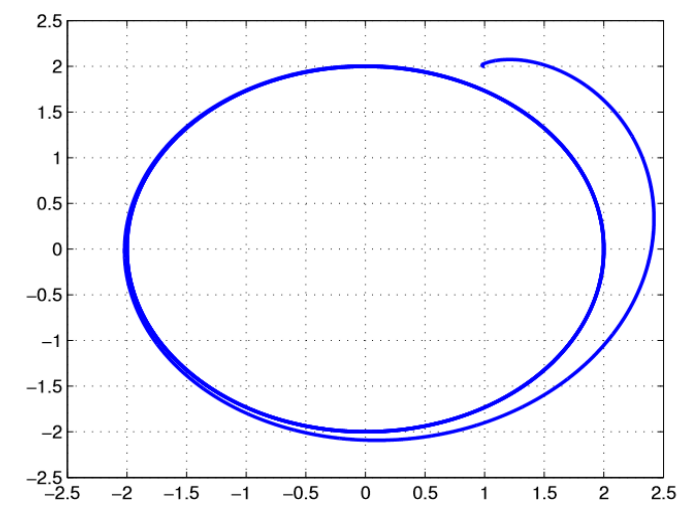

Fig. 3. A level tracking with continuous measurement. The initial condition: $(1,2)$, the boundary to be tracked: $x^{2}+y^{2}=4$.

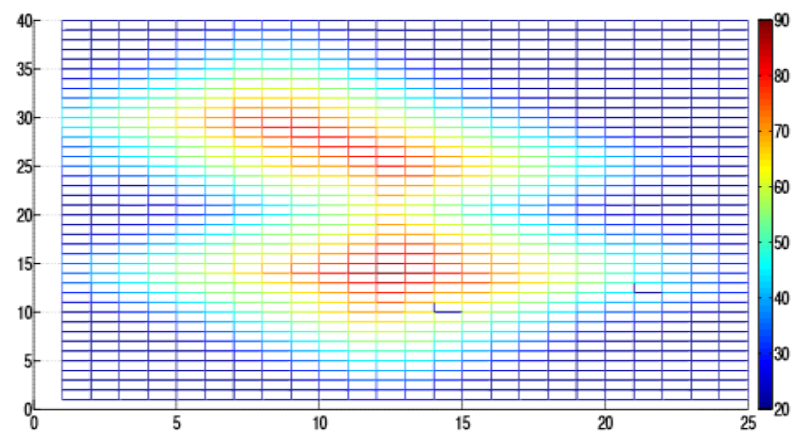

Fig. 4. The level set data map

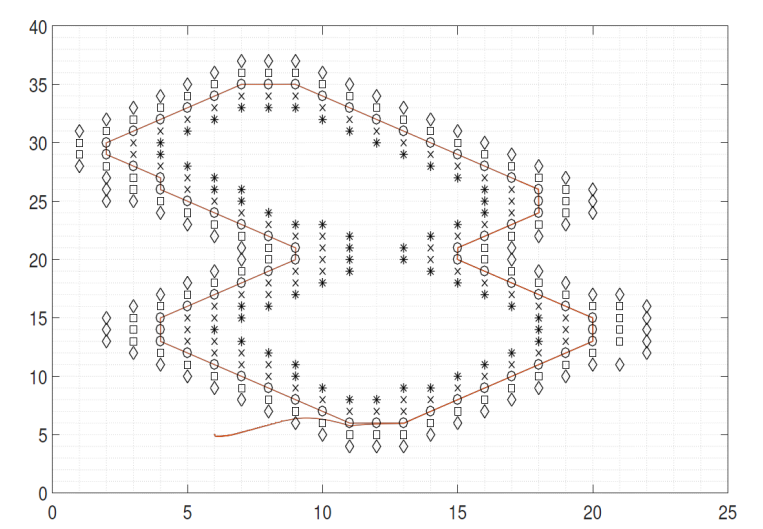

$\bigcirc: \gamma(x, y)=50, \quad \mathrm{x}: *: \gamma(x, y)=60, \square: \gamma(x, y)=45, \diamond: \gamma(x, y)=55$, $\gamma(x, y)=40$. The inside of the level set $\gamma(x, y)=60$, is set to values higher than 60 and the outside of the level set $\gamma(x, y)=40$ is set to values lower than 40

Fig. 5. A level set tracking with discontinuous measurement. The initial condition: $(6,5)$

set to $k_{1}=-2, k_{2}=-3$, and $\bar{b}=10$, and the DOB parameters are set to $\bar{g}=1, a_{2}=2, a_{1}=0.2$, and $\tau=0.0001$. Fig. 3 shows the simulation result. As shown in Fig. 3, the agent follows the trajectory $x^{2}+y^{2}=4$ successfully due to the DOB based boundary tracking control.

\subsection{Discrete measurement case}

In this subsection, it is shown that the proposed controller can also solve a boundary tracking problem even when the boundary data is given in a discrete manner. For the purpose, a data map is generated in a way that function values of $\gamma(x, y)$ are assigned at each integer points over $0<x<25,0<y<40$. See Fig. 4. The problem is to make the agent follow the level set $\gamma(x, y)=50$.

As seen in Fig. 5, the agent controlled by the proposed DOB based control follows the level set $\gamma(x, y)=50$ successfully although the data are given discretely. In order to get the function values of $\gamma(x, y)$ at non-integer points, interpolation is used.

\section{Application to Environment Monitoring}

Pollution in environment caused due to spread of abrupt release of contaminants in a medium is a potential high risk to society. Leakage of harmful gas, nuclear radiation from unshielded devices, oil spill in oceans, spread of ash clouds from volcanic eruptions are few such important examples. In all these hostile situations, the environmental monitoring and control authorities have to assess the spread of spatial phenomena in a safe manner, such that appropriate countermeasures may be taken in sequel. In this section, we demonstrate the use of proposed methodology in identifying the spread of ash clouds from volcanic eruption. The assumption here is that the Unmanned Air Vehicle (UAV) is equipped with a suitable sensor payload which can fly and take measurements within ash cloud. Numerical Atmospheric-dispersion Modeling Environment (NAME), is a Lagrangian dispersion model used by UK Met Office, in which a several model 'particles' are initialized and evolved through the computational atmosphere. The numerical weather prediction models and parameters such as plume height, mass eruption rate and vertical distribution of ash become the driving meteorology for the NAME model [16]. In NAME, the particles are advanced in space due to the resolved wind and due to a random motion representing the effects of unresolved motions. For further details see [17] and [9] in which the data has been used to validate a sub-optimal boundary tracking control law.

The data generated by NAME over a 300 square kilometers of spatial region at a constant height is considered in this study and is depicted in Fig. 6. Note that in the simulations the measurement units are scaling of the original units and the aspect ratio is not retained. The idea here is to validate the performance of the proposed boundary tracking concept using realistic data, and henceforth the capability of vehicle to cover the entire region in one mission is relaxed. The main point here is that once the vehicle is deployed over the region, the only 
measurement available for the vehicle to steer over the region autonomously is only the instantaneous density measurement value of the contaminant at that location.

The agent dynamics in (1) together with the control law in (6) are simulated in a MATLAB(2015b)/SIMULINK simulation environment. The integration scheme followed is ode1(Euler) and the step size is fixed at 0.001. During the simulations, it is assumed that the agent only measure the local intensity of volcanic ash. This is realized by passing the instantaneous position of the vehicle $(x(t)$, $y(t)$ ) to the numerical atmospheric-dispersion modeling environment (NAME) and corresponding contaminant density measurement $\gamma(x(t), y(t))$ is obtained. The velocity of the vehicle $V$ in (1) is fixed at 1 unit/min which corresponds to a speed of approximately $5 \mathrm{~m} / \mathrm{sec}$.

In the simulation framework, the agent is initialized at location $(84,142)$, which is represented as a 'red' circle in Fig. 6. The steering angle $\theta$ is initially fixed at $\pi / 3$ rads. The curve in black color in Fig. 6 represents the trajectory that the vehicle has taken autonomously and shows the goodness of the tracking performance of the proposed boundary tracking control law.

The tracking performance is poor in the circle in Fig. sn6. This is mainly due to a sharp change in the gradient of the contour and the requirements of a sharp turn which make Assumption 2 violated.

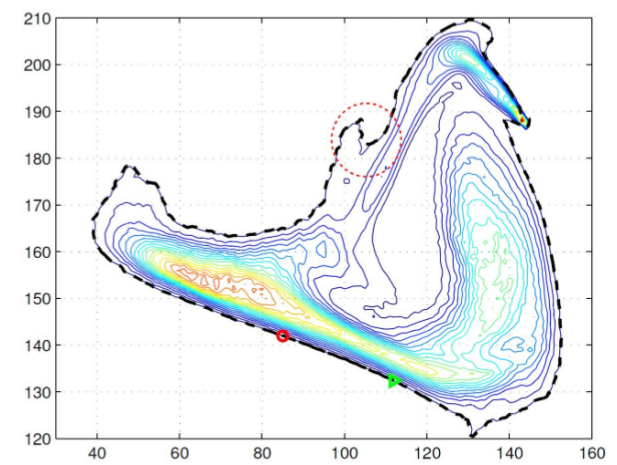

Fig. 6. Volcanic ash dispersion data and boundary tracking performance (thick dashed line). The initial condition: $(84,142)$

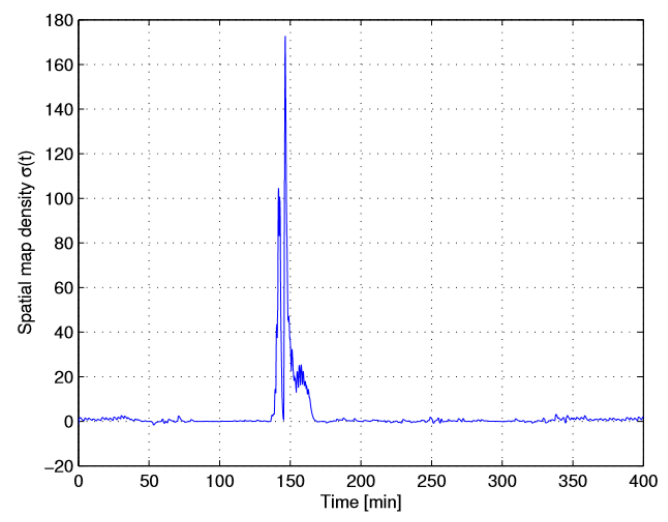

Fig. 7. The tracking error $\sigma(t)=\gamma(x, y)-\gamma^{*}$ over time
Fig. 7 shows the tracking error $\sigma(t)=\gamma(x, y)-\gamma^{*}$. The large error near $t=150$ corresponds to that in the circle in Fig. 6. This is mainly due to large variation of the measured boundary data which makes Assumption 2 fail to hold. Except for around $t=150$, the tracking performance is quite good in view of Fig. 7.

Fig. 8 is the corresponding control input. Figs. 9-11 show the states of the Q-filters. As seen in Figs. 9-11, the behavior of the Q-filters states are similar to that of bang bang control. This is because the interpolation is used in obtaining the measured data $\gamma(x, y)$ when $\gamma(x, y)$ is known at $(x, y)$. This interpolated $\gamma(x, y)$ can make

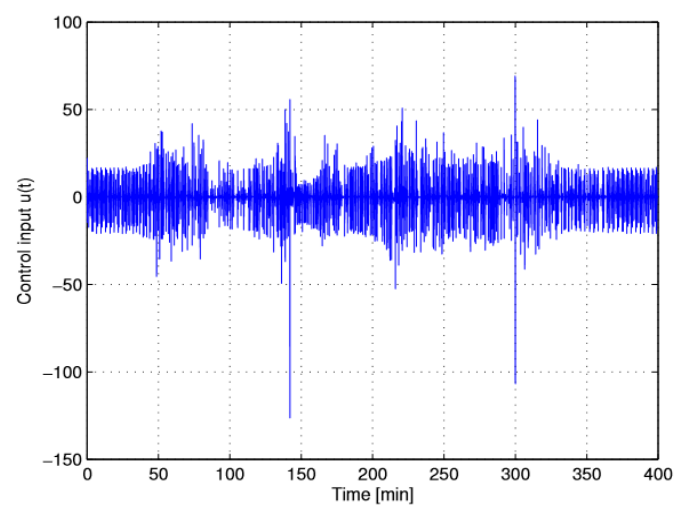

Fig. 8. The control input $u(t)$

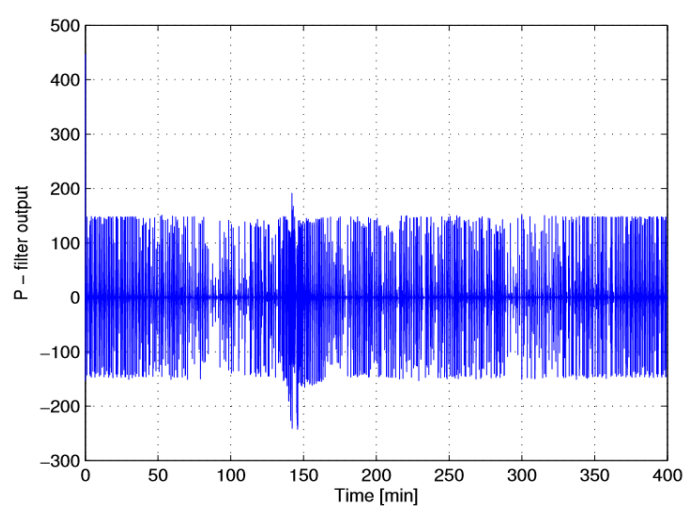

Fig. 9. Output of the filter $Q_{B}$

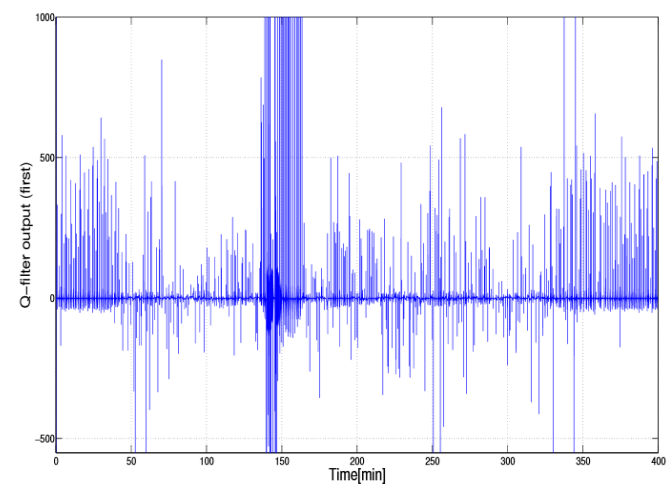

Fig. 10. Output of the filter $Q_{A}: q_{1}$, estimate of $z$. 


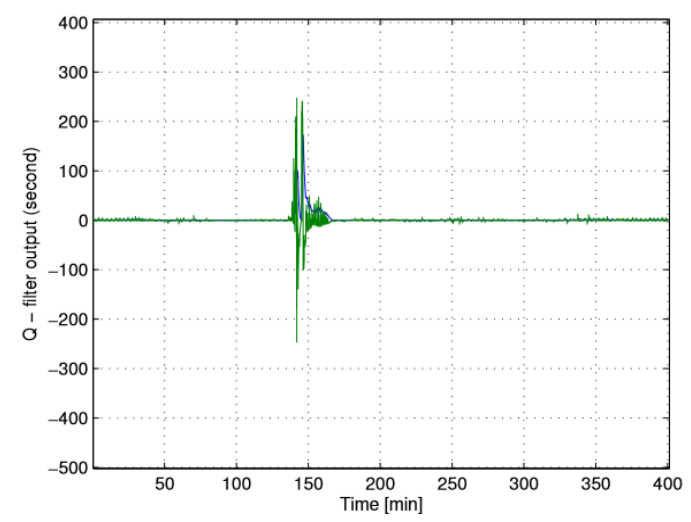

Fig. 11. Output of the filter $Q_{A}: q_{2}$, estimate of $\dot{z}$.

sharp gradient, which can result in bang bang control like behavior of the Q-filter states.

\section{Conclusion}

This paper presented a boundary tracking of an agent. To this end, the problem is reformulated into robust control design for uncertain double integrators. Then, a disturbance observer (DOB) based control is designed to stabilize the uncertain double integrator. In order to demonstrate the effectiveness of the proposed control, the designed DOB based control is applied to two simple examples; boundaries are given in a continuous and discrete manner. Finally, it is shown that the proposed control can be employed for environmental monitoring. For the purpose, synthetic volcanic eruption dispersion data is used as boundary information. It turned out that the proposed control leads to successful tracking performance for the realistic boundary data.

\section{Acknowledgements}

This research was supported by Basic Science Research Program through the National Research Foundation of Korea (NRF) funded by the Ministry of Education (NRF2015R1D1A1A01060588) and by the Human Resources Development of the Korea Institute of Energy Technology Evaluation and Planning (KETEP) grant funded by the Korea government Ministry of Trade, Industry \& Energy (20154030200720).

\section{References}

[1] C. T. Hardin, X. Cui, R. K. Ragade, J. H. Graham, and A.S. Elmaghraby. A modified particle swarm algorithm for robotic mapping of hazardous environments. In Proceedings of the World Automation
Congress, 2004.

[2] C. M. Hsieh, Z. Jin, D. Marthaler, B. Q. Nguyen, D. J. Tung, A. L. Bertozzi, and R. M. Murray. Experimental validation of an algorithm for cooperative boundary tracking. In Proceedings of American Control Conference, New York, 2005.

[3] D. Marthaler and A. L. Bertozzi. Tracking environmental level sets with autonomous vehicles. In In Recent Developments in Cooperative Control and Estimation, Kluwer Academic Publishers, 2004.

[4] J. Clark and R. Fierro. Cooperative hybrid control of robotic sensors for perimeter detection and tracking. In Proceedings of American Control Conference, Portland, 2005.

[5] D. Baronov and J. Baillieul. Autonomous vehicle control for ascending/descending along a potential field with two applications. In Proceedings of American Control Conference, 2008.

[6] J. Cochran and M. Krstic. Source seeking with a nonholonomic unicycle without position measurements and with tuning of angular velocity part $\mathrm{i}$ : Stability analysis. In Proceedings of 46th IEEE Conference on Decision and Control, New Orleans, 2007.

[7] L. Brinon-Arranz, A. Seuret, and C. Canudas de Wit. Collaborative estimation of gradient direction by a formation of AUVs under communication constraints. In Proceedings of 50th IEEE Conference on Decision and Control and European Control Conference, Orlando, 2011.

[8] C. Canudas de Wit, F. Garin, R. Fabbiano, P. Rouchon, and A. Rousseau. Source localization using poisson integrals. In In Proceedings of 3rd IFAC Workshop on distributed estimation and control in networked systems (NecSys'12), 2012.

[9] P. P. Menon, C. Edwards, Y. B. Shtessel, D. Ghose, and J. Haywood. Boundary tracking using a suboptimal sliding mode algorithm. In the 53rd Proceeding of IEEE Conference on Decision and Control, 2014.

[10] P. P. Menon and D. Ghose. Simultaneous source localisation and boundary mapping for contaminants. In Proceedings of American Contol Conference, Washington D. C., 2013.

[11] K. N. Krishnanand and D. Ghose. Detection of multiple source locations using a glowworm metaphor with applications to collective robotics. In Pro-ceedings of IEEE Swarm Intelligence Symposium (SIS 2005), Pasadena, California, 2005.

[12] P. P. Menon and D. Ghose. 3-dimensional boundary mapping. In Proceedings of American Control Conference, Montreal, 2012.

[13] J. Back and H. Shim. Adding robustness to nominal output-feedback controllers for uncertain nonlinear systems: A nonlinear version of disturbance observer. Automatica, 44:2528-2537, 2008.

[14] J. Back and J.-S. Kim. A disturbance observer based 
practical coordinated tracking controller for uncertain heterogeneous multi-agent systems. Int. J. Robust and Nonlinear Control, 25:2254-2278, 2015.

[15] H. K. Khalil. Nonlinear Systems, Third Ed. PrenticeHall, Upper Saddle River, NJ, 2002.

[16] S. J. Leadbetter and M. C. Hort. Volcanic ash hazard climatology for an eruption of hekla volcano, iceland. J. Volcanol. Geotherm. Res., 199:230-241, 2011.

[17] H. N. Webster, D. J. Thomson, B. T. Johnson, I. P. C. Heard, K. Turnbull, F. Marenco, N. I. Kristiansen, J. Dorsey, A. Minikin, B. Weinzierl, U. Schumann, R. S. J. Sparks, S. C. Loughlin, M. C. Hort, S. J. Leadbetter, B. J. Devenish, A. J. Manning, C. S. Witham, J. M. Haywood, and B. W. Golding. Operational prediction of ash concentrations in the distal volcanic cloud from the 2010 Eyjafyallajökull eruption. J. Geophys. Res., 117, 2012.

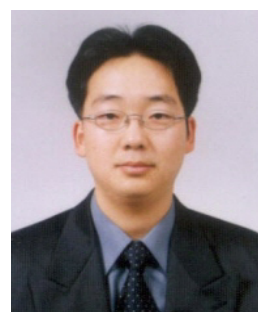

Jung-Su Kim He received BS., MS., and $\mathrm{Ph} . \mathrm{D}$ degree in electrical engineeering from Korea University. Since 2009 he has been with Dept. of Electrical and Information Engineering, Seoul Nat'l University of Science and Technology. His research interest includes MPC and its application to energy systems.

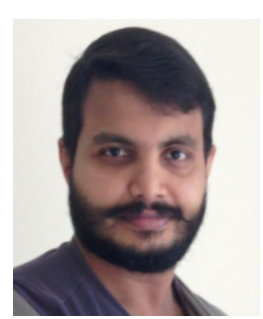

Prathyush P. Menon Dr. Prathyush Menon was awarded a PhD in Control Engineering from Leicester University in 2007. He is currently a Senior Lecturer at the University of Exeter, UK. His research interests are broadly in the areas of optimization, robust and nonlinear control and observation theory, and their application to aerospace systems.

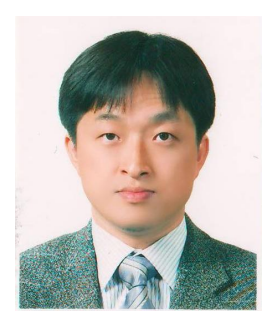

Juhoon Back He received his B.Sc. and M.Sc. degrees in Mechanical Design and Production Engineering from Seoul National University, in 1997 and 1999, respectively. $\mathrm{He}$ received his Ph.D. degree from the School of Electrical Engineering and Computer Science, Seoul National University in 2004. From 2005 to 2006, he worked as a research associate at Imperial College London, UK. Since 2008 he has been with Kwangwoon University, Korea, where he is currently an associate professor. His research interests include control system theory and design, renewable energy systems, and multi agent systems.

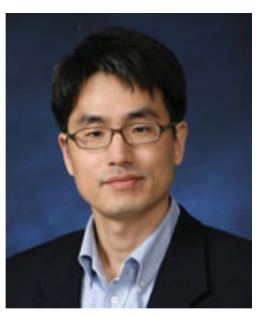

Hyungbo Shim He received his BS, $\mathrm{MS}$, and $\mathrm{PhD}$ degrees from Seoul National University, Korea, in 1993, 1995, and 2000, respectively. From 2000 to 2001, he was a postdoctoral researcher at the Center for Control Engineering and Computation, University of California, Santa Barbara. In 2002, he joined the faculty of the Division of Electrical and Computer Engineering, Hanyang University, Seoul, Korea. From 2003, he has been with the Department of Electrical and Computer Engineering at Seoul National University, Korea, where he is currently an associate professor. His research interests include analysis and control of nonlinear systems. He had been an associate editor in European Journal of Control, and is currently for IEEE Transactions on Automatic Control and International Journal of Robust and Nonlinear Control 